\title{
Coloring Uniform Hypergraphs With Few Edges*
}

\author{
A.V. Kostochka, ${ }^{1,2}$ M. Kumbhat ${ }^{1}$ \\ ${ }^{1}$ Department of Mathematics, University of Illinois, Urbana, Illinois 61801; \\ e-mail: kostochk@math.uiuc.edu; kumbhat2@uiuc.edu \\ ${ }^{2}$ Institute of Mathematics, Novosibirsk, Russia
}

Received 16 January 2008; accepted 3 October 2008; received in final form 14 November 2008

Published online 27 July 2009 in Wiley InterScience (www.interscience.wiley.com).

DOI 10.1002/rsa.20284

ABSTRACT: A hypergraph is $b$-simple if no two distinct edges share more than $b$ vertices. Let $m(r, t, g)$ denote the minimum number of edges in an $r$-uniform non-t-colorable hypergraph of girth at least $g$.

Erdős and Lovász proved that

$$
\begin{aligned}
m(r, t, 3) & \geq \frac{t^{2(r-2)}}{16 r(r-1)^{2}} \\
\text { and } \quad m(r, t, g) & \leq 4 \cdot 20^{g-1} r^{3 g-5} t^{(g-1)(r+1)} .
\end{aligned}
$$

A result of Szabó improves the lower bound by a factor of $r^{2-\epsilon}$ for sufficiently large $r$. We improve the lower bound by another factor of $r$ and extend the result to $b$-simple hypergraphs. We also get a new lower bound for hypergraphs with a given girth. Our results imply that for fixed $b, t$, and $\epsilon>0$ and sufficiently large $r$, every $r$-uniform $b$-simple hypergraph $\mathcal{H}$ with maximum edge-degree at most $t^{r} r^{1-\epsilon}$ is $t$-colorable. Some results hold for list coloring, as well. @ 2009 Wiley Periodicals, Inc. Random Struct. Alg., 35, 348-368, 2009

Keywords: hypergraph coloring; maximum degree; edge-degree; small size

\section{INTRODUCTION}

Let $\mathcal{H}$ be a hypergraph with vertex set $V(\mathcal{H})$ and edge set $E(\mathcal{H})$. The degree of a vertex $v$ is the number of edges containing $v$. Similarly, the degree of an edge $e$ is the number of edges intersecting $e$. We will denote by $\Delta(\mathcal{H})$ the maximum degree of vertices in $\mathcal{H}$. A cycle of length $k$ in a hypergraph $\mathcal{H}$ is an alternating cyclic sequence $e_{0}, v_{0}, e_{1}, v_{1}, \ldots, e_{k-1}, v_{k-1}, e_{0}$

Correspondence to: A.V. Kostochka

*Supported by: NSF Grant DMS-0650784; Grant 06-01-00694 of the Russian Foundation for Basic Research. (C) 2009 Wiley Periodicals, Inc. 
of distinct edges and vertices in $\mathcal{H}$ such that $v_{i} \in e_{i} \cap e_{i+1}$ for all $i$ modulo $k$. The girth of a hypergraph is the length of its shortest cycle. The distance between two vertices is the number of edges in a shortest path connecting them. A hypergraph of girth at least three is also called simple. Let $m(r, t, g)$ denote the smallest number of edges in an $r$-uniform hypergraph with girth at least $g$ and chromatic number at least $t+1$. In their seminal article [2], Erdôs and Lovász gave the upper bound

$$
m(r, t, g) \leq 4 \cdot 20^{g-1} r^{3 g-5} t^{(g-1)(r+1)}
$$

for all $g$ and the lower bound

$$
m(r, t, 3) \geq \frac{t^{2(r-2)}}{16 r(r-1)^{2}}
$$

for simple hypergraphs. The ratio of the upper bound to the lower bound for simple hypergraphs is only $r^{7}$. The bound (2) was derived from the following famous result.

Theorem 1 [2]. If $t, r \geq 2$, then every $r$-uniform hypergraph $\mathcal{H}$ with maximum degree of its edges at most $\frac{1}{4} t^{r}$ is $t$-colorable. In particular, if the maximum vertex degree of $\mathcal{H}$ is at most $\frac{1}{4} t^{r} r^{-1}$, then $\mathcal{H}$ is $t$-colorable.

To derive the bound, they used an interesting trick of trimming. We discuss trimming in Subsection 3.1.

Szabó [3] refined the second part of the bound of Theorem 1 for simple hypergraphs as follows.

Theorem 2. If $t \geq 2$ and $\epsilon>0$ are fixed and $r$ is sufficiently large, then every $r$-uniform simple hypergraph $\mathcal{H}$ with maximum degree at most $t^{r} r^{-\epsilon}$ is $t$-colorable.

Actually, Szabó proved the theorem only for $t=2$, since that was what he needed for his applications, but the technique works for any fixed $t$. Again, applying trimming and this theorem, one easily gets that for fixed $t$ and $\epsilon$ and large $r$,

$$
m(r, t, 3) \geq \frac{t^{2 r}}{r^{1+\epsilon}}
$$

In this article, we consider simple and so called $b$-simple hypergraphs. A hypergraph $\mathcal{H}$ is $b$-simple if $\left|e \cap e^{\prime}\right| \leq b$ for every distinct $e, e^{\prime} \in E(\mathcal{H})$. Sometimes, $b$-simple hypergraphs are called partial Steiner systems. A 1-simple hypergraph is a simple hypergraph.

The main result of this article (we state it in the next section) says that for fixed $t \geq 2$ and $\epsilon>0$ and sufficiently large $r$, if a simple $r$-uniform hypergraph $\mathcal{H}$ cannot be colored with $t$ colors, then either it has a vertex of degree greater than $r t^{r}$, or there are "many" vertices of degree greater than $t^{r} r^{-\epsilon}$. This will improve the bound (3) by a factor of $r$. Our result also yields an improvement of the edge-degree version of Theorem 1 for simple hypergraphs as follows.

Theorem 3. If $b \geq 1, t \geq 2$, and $\epsilon>0$ are fixed and $r$ is sufficiently large, then every $r$-uniform b-simple hypergraph $\mathcal{H}$ with maximum edge-degree at most $t^{r} r^{1-\epsilon}$ is $t$-colorable. 
The theorem holds also for list colorings. To keep proofs easier to read, we give the proof for ordinary colorings and comment at the end of the article how to adapt the proofs to list coloring.

Let $f(r, t, b)$ denote the smallest possible number of edges in an $r$-uniform $b$-simple hypergraph that is not $t$-colorable. From our main result we deduce that for fixed $t, b$, and $\epsilon>0$ and sufficiently large $r$,

$$
f(r, t, b) \geq \frac{t^{r(1+1 / b)}}{r^{\epsilon}} .
$$

It turns out that in terms of $r$, the bound cannot be improved by more than a polynomial factor. Using the Erdôs-Lovász technique [2] for proving (2), we show that for large $r$,

$$
f(r, t, b) \leq 40 t^{2}\left(t^{r} r^{2}\right)^{1+1 / b} .
$$

We also use our main result and trimming to derive the following lower bounds on $m(r, t, g)$ for arbitrary fixed $g$ (in [2], the bound was only for $g=3$ ):

$$
m(r, t, 2 s+1) \geq \frac{t^{r(1+s)}}{r^{\epsilon}},
$$

if $r$ is large in comparison with $t, s$, and $1 / \epsilon$.

The structure of the article is as follows. In the next section we prove the main result. In Section 3, lower bounds on the size of non- $t$-colorable hypergraphs are given. In Section 4 , bound (5) is derived. We conclude the article with some comments. In particular, we comment on list colorings of hypergraphs.

\section{COLORING HYPERGRAPHS WITH BOUNDED EDGE DEGREES}

Szabó's theorem says that for large $r$, every $r$-uniform simple hypergraph with the degree of each vertex at most $t^{r} r^{-\epsilon}$ is $t$-colorable. Our result extends the conclusion to $r$-uniform simple (and $b$-simple) hypergraphs in which the degree of each edge is at most $t^{r} r^{1-\epsilon}$.

A vertex $v$ of $\mathcal{H}$ is low, if $\operatorname{deg}(v) \leq t^{r} r^{-\epsilon}$ and high otherwise. For an edge $e$, let $L(e)$ [respectively, $H(e)]$ be the set of low (respectively, high) vertices in $e$. An edge $e$ is light, if $|H(e)| \leq 0.5 r$ and heavy otherwise.

For a given $\epsilon>0$, an $r$-uniform hypergraph $\mathcal{H}$ is $(t, \epsilon)$-sparse if

$$
\Delta(\mathcal{H}) \leq t^{r} r \text {, and }
$$

every vertex of $\mathcal{H}$ is in at most $t^{r} / r^{\epsilon}$ heavy edges.

Our main result is the following.

Theorem 4. If $b \geq 1, t \geq 2$, and $\epsilon>0$ are fixed and $r$ is sufficiently large, then every $r$-uniform b-simple $(t, \epsilon)$-sparse hypergraph $\mathcal{H}$ is $t$-colorable.

To derive Theorem 3 from our main result, we observe that for sufficiently large $r$, every not $(t, 0.5 \epsilon)$-sparse hypergraph $\mathcal{H}$ has an edge of degree greater than $t^{r} r^{1-\epsilon}$. This is trivial if (7) does not hold. Suppose now that (8) does not hold, in particular that some edge $e$ in $\mathcal{H}$ is heavy. Then the sum of degrees of vertices in $e$ is greater than $0.5 r t^{r} r^{1-0.5 \epsilon}$. Since every edge $e^{\prime} \neq e$ contributes at most $b$ to this sum, $e$ itself contributes $r$, and $r^{0.5 \epsilon}>4 b$, the degree of $e$ in $\mathcal{H}$ is greater than $t^{r} r^{1-\epsilon}$. This proves Theorem 3 (modulo Theorem 4). 


\subsection{Szabó's Approach and the Structure of the Proof}

We follow the ideas of Szabó [3]. He used the following lemma of Beck [1], who in turn used the Local Lemma.

Lemma 5 [Beck]. Let $X$ be a finite set and $B_{1}, B_{2}, \ldots, B_{s}$ be not necessarily distinct subsets of $X$ with $\left|B_{i}\right| \geq r$. For every $i$, let $f_{i}: B_{i} \rightarrow\{1,2, \ldots, t\}$ be a given $t$-coloring of $B_{i}$. If

$$
\sum_{i: p \in B_{i}}\left(1-\frac{1}{r}\right)^{-\left|B_{i}\right|} t^{-\left|B_{i}\right|} \leq \frac{1}{r}
$$

for every $p \in X$, then there exists a $t$-coloring $f: X \rightarrow\{1,2, \ldots, t\}$ such that $\left.f\right|_{B_{i}} \neq f_{i}$.

Szabó's idea of the proof is the following. Let $\mathcal{H}$ be an $r$-uniform simple hypergraph satisfying the conditions of his theorem. Szabó starts from a $t$-coloring of vertices of $\mathcal{H}$ where each vertex is colored with a color uniformly at random chosen from the set $\{1, \ldots, t\}$ independently from all other vertices. He considers a special set of so called configurations that are pairs $\left(B_{i}, f_{i}\right)$, where $B_{i} \subseteq V(\mathcal{H})$ and $f_{i}$ is a given $t$-coloring of $B_{i}$. The meaning of configurations, is that they are bad situations that may cause some edges to become monochromatic after special recolorings in the future. He proved that

a. if $f$ is any (not necessarily proper) $t$-coloring of $V(\mathcal{H})$ and none of his configurations occurs, then some vertices of $\mathcal{H}$ can be recolored so that the resulting $t$-coloring of $\mathcal{H}$ is proper;

b. Inequality (9) holds for every $p \in V(\mathcal{H})$.

Together with Lemma 5, this yields that $\mathcal{H}$ has a proper $t$-coloring. Observe that each configuration $B \subseteq V(\mathcal{H})$ contributes to the sum in (9) the amount $\left(1-\frac{1}{r}\right)^{-|B|} t^{-|B|}$, and we will call this expression the contribution of $B$. To prove that (9) holds, for every "bad" configuration $B \subseteq V(\mathcal{H})$, Szabó estimated its contribution.

We will use the same scheme with somewhat changed rules of recoloring and somewhat different configurations.

Another idea of Szabó is that in each edge $e$ of $\mathcal{H}$ he chooses a subset $R(e)$ such that later, if $e$ is monochromatic, then he tries to recolor only vertices in $R(e)$ and does not touch other vertices. This choice allows to decrease the number of "bad" configurations whose contributions we need to estimate. The structure of our proof is the following. In the next subsection, we construct a subset $R(e)$ of each edge $e$. Later, if $e$ becomes monochromatic, we will try to recolor only vertices in $R(e)$. In Subsection 2.3, we give the main proof assuming that we have some bounds on the contributions of "bad" configurations. In Subsections 2.4 and 2.5, we prove these bounds on contributions.

\subsection{Choosing $R(e)$}

Lemma 6. Let $k \leq r / 3$. Then in every light edge $e$, we can choose a k-element set $R(e) \subseteq L(e)$ so that for each low vertex $v$,

$$
|\{e: v \in R(e)\}| \leq \frac{t^{r}}{r^{\epsilon}} \frac{4 k}{r} .
$$


Proof. Consider the bipartite graph $G[X, Y]$, where $X$ is the set of light edges in $\mathcal{H}, Y$ is the set of low vertices in $\mathcal{H}$, and $x y \in E(G)$ if and only if edge $x$ contains vertex $y$ in $\mathcal{H}$. By the definition of light edges, each vertex in $X$ has degree in $G$ at least $r / 2$. By the definition of low vertices, $d_{G}(v) \leq t^{r} / r^{\epsilon}$ for every $v \in Y$. Let $G_{1}$ be the graph obtained from $G$ by splitting every vertex $v \in Y$ into $\left\lceil 2 d_{G}(v) / r\right\rceil$ vertices, each with degree at most $\lceil r / 2\rceil$.

Let $G_{2}$ be obtained from $G_{1}$ by deleting some edges so that the degree of every vertex $x \in X$ becomes $\lceil r / 2\rceil$. By Konig's theorem, there exists a proper edge-coloring $\phi$ of $G_{2}$ with $\lceil r / 2\rceil$ colors. Let $G_{3}$ be the subgraph of $G_{2}$ formed by the edges with colors $\{1,2, \ldots k\}$ in $\phi$. Finally, let $G_{4}$ be obtained from $G_{3}$ by gluing back all the split vertices in $Y$. By construction, $G_{4}$ is a spanning subgraph of $G$, and the degree of every vertex $x \in X$ in $G_{4}$ is exactly $k$. The degree in $G_{4}$ of every vertex $v \in Y$ is at most

$$
k\left\lceil 2 d_{G}(v) / r\right\rceil \leq k\left\lceil\frac{t^{r}}{r^{\epsilon}} \frac{2}{r}\right\rceil .
$$

The last expression for large $r$ does not exceed the RHS of (10).

For every edge $e$ in $\mathcal{H}$, let $R(e)$ be the set of vertices adjacent to vertex $e$ in $G_{4}$. By the properties of $G_{4}$, the lemma holds for these $R(e)$.

Lemma 7. Let $k \leq r / 3$. Then in every heavy edge $e$, we can choose a k-element set $R(e) \subseteq H(e)$ so that for each heavy vertex $v$,

$$
|\{e: v \in R(e)\}| \leq \frac{t^{r}}{r^{\epsilon}} \frac{4 k}{r} .
$$

Proof. By (8), every vertex is in at most $t^{r} r^{-\epsilon}$ heavy edges. We essentially repeat the proof of Lemma 6, only replacing light edges with heavy and low vertices with high ones.

\subsection{Configurations and the Main Proof}

We start from a random $t$-coloring $f$ of vertices of $\mathcal{H}$ where each vertex $v$ is colored with a color $f(v)$ uniformly at random chosen from the set $\{1, \ldots, t\}$ independently from all other vertices.

2.3.1. Configurations of Type 1. A configuration of Type $1, \mathcal{C}\left(j, m, m^{\prime}, m_{1}, \ldots, m_{m}\right)$, with parameters $j, m, m^{\prime}, m_{1}, \ldots, m_{m}$ consists of $1+m+m^{\prime}+\left(m_{1}+\cdots+m_{m}\right)$ (not necessarily distinct) edges $D, B_{1}, \ldots, B_{m^{\prime}}, C_{1}, \ldots, C_{m}, A_{1,1}, \ldots, A_{1, m_{1}}, A_{2,1}, \ldots, A_{m, m_{m}}$ arranged and colored (Fig. 1) so that:

$\left(\alpha_{1}\right)$ There are $m^{\prime}$ distinct vertices $b_{1}, \ldots, b_{m^{\prime}}$ in $H(D)$ such that $b_{i} \in R\left(B_{i}\right)$ for $i=1, \ldots, m^{\prime}$.

$\left(\alpha_{2}\right)$ There are $m$ distinct vertices $c_{1}, \ldots, c_{m}$ in $L(D)$ such that $c_{i} \in R\left(C_{i}\right)$ for $i=1, \ldots, m$.

$\left(\alpha_{3}\right)$ All $B_{1}, \ldots, B_{m^{\prime}}, C_{1}, \ldots, C_{m}$ are distinct.

$\left(\alpha_{4}\right)$ All vertices in $D-\left\{b_{1}, \ldots, b_{m^{\prime}}, c_{1}, \ldots, c_{m}\right\}$ are colored with color $j+1$ (modulo $t$ ).

$\left(\alpha_{5}\right)$ All vertices in $B_{1}, \ldots, B_{m^{\prime}}$ are colored with $j$.

$\left(\alpha_{6}\right)$ For $i=1, \ldots, m, H\left(C_{i}\right)$ contains $m_{i}$ distinct vertices $a_{i, 1}, \ldots, a_{i, m_{i}}$ such that $a_{i, i^{\prime}} \in$ $R\left(A_{i, i^{\prime}}\right)$ for all $i^{\prime}=1, \ldots, m_{i}$.

$\left(\alpha_{7}\right)$ Vertex $a_{i_{1}, i_{1}^{\prime}}$ may coincide with $a_{i_{2}, i_{2}^{\prime}}$, when $i_{1} \neq i_{2}$, in which case $A_{i_{1}, i_{1}^{\prime}}$ should coincide with $A_{i_{2}, i_{2}^{\prime}}$. If $a_{i_{1}, i_{1}^{\prime}} \neq a_{i_{2}, i_{i}^{\prime}}$, then $A_{i_{1}, i_{1}^{\prime}} \neq A_{i_{2}, i_{2}^{\prime}}$.

$\left(\alpha_{8}\right)$ For every $i=1, \ldots, m$ all vertices in $C_{i}-\left\{a_{i, 1}, \ldots, a_{i, m_{i}}\right\}$ are colored with $j$.

$\left(\alpha_{9}\right)$ All vertices in all $A_{1,1}, \ldots, A_{1, m_{1}}, A_{2,1}, \ldots, A_{m, m_{m}}$ are colored with $j-1$ (modulo $t$ ). 


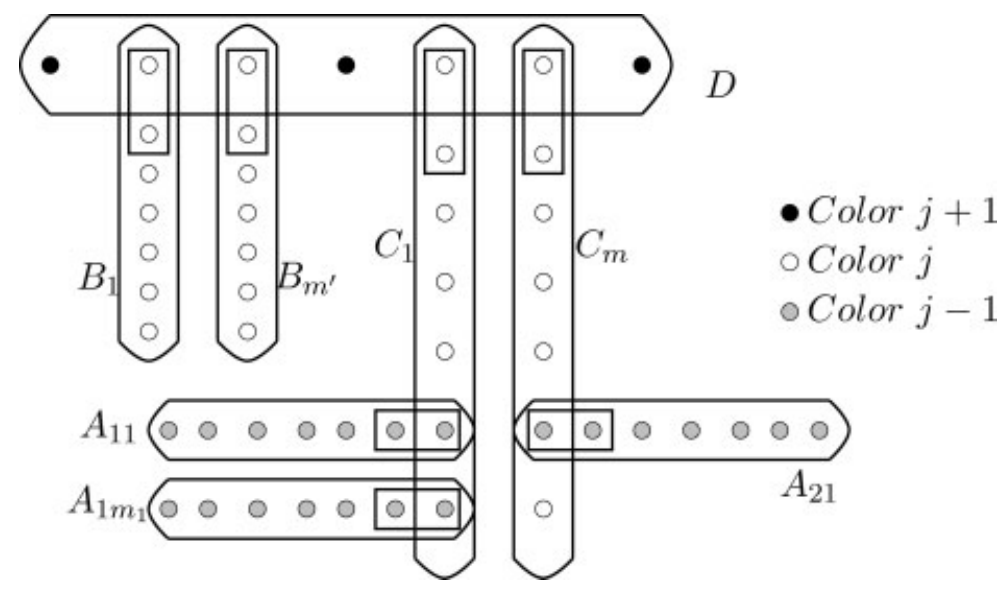

Fig. 1. An example of a Configuration of Type 1.

Comments: Since $b_{1}, \ldots, b_{m^{\prime}} \in H(D)$, each of $B_{1}, \ldots, B_{m^{\prime}}$ is a heavy edge. For the same reason, each of $A_{1,1}, \ldots, A_{1, m_{1}}, A_{2,1}, \ldots, A_{m, m_{m}}$ is heavy. Similarly, each of $C_{1}, \ldots, C_{m}$ is a light edge.

In a configuration of Type 1, edge $D$ is called the leading edge, edges $B_{1}, \ldots, B_{m^{\prime}}$ are type $B$ edges, edges $C_{1}, \ldots, C_{m}$ are type $C$ edges. Vertices $b_{1}, \ldots, b_{m^{\prime}}$ and $c_{1}, \ldots, c_{m}$ are special in $\mathcal{C}$. The size of a configuration is the cardinality of the union of its edges.

Let $k=\left\lceil\frac{20}{\epsilon}\right\rceil$. In the next subsection, we will prove that for every vertex $p$ in $\mathcal{H}$, the total contribution of configurations of Type 1 containing $p$ such that at least one of $m, m^{\prime}, m_{1}, \ldots, m_{m}$ exceeds $k$ is $o(1 / r)$.

2.3.2. Configurations of Type 2a. There is a heavy edge $B$ such that for each vertex $b \in R(B)$ there is a configuration $\mathcal{C}_{b}$ of Type 1 with $m=0$ and $m^{\prime} \leq k$ such that $b$ is special and $B$ is an edge of type $B$ in $\mathcal{C}_{b}$.

2.3.3. Configurations of Type $2 b$. There is a light edge $C$ such that for each vertex $c \in R(C)$ there is a configuration $\mathcal{C}_{c}$ of Type 1 with each of $m^{\prime}, m, m_{1}, \ldots, m_{m}$ at most $k$ such that $c$ is special and $C$ is an edge of type $C$ in $\mathcal{C}_{c}$ (Fig. 2).

In Subsection 2.5, we prove that for every vertex $p$ in $\mathcal{H}$, the total contribution of configurations of Types $2 \mathrm{a}$ and $2 \mathrm{~b}$ containing $p$ is $o(1 / r)$. These facts together with Lemma 5 yield that there exists a $t$-coloring $f^{\prime}$ avoiding configurations of Type 1 with at least one of $m^{\prime} m, m_{1}, \ldots, m_{m}$ exceeding $k$ and also avoiding all configurations of Type $2 \mathrm{a}$ and $2 \mathrm{~b}$. This coloring $f^{\prime}$ might have monochromatic edges, but we shall see that we can recolor some of the vertices and get a proper $t$-coloring.

First recoloring: Since configurations of Type 2a do not appear in $f^{\prime}$, for every heavy monochromatic edge $B$ [say, of color $j(B)$ ], there exists a vertex $b(B) \in R(B)$ such that there is no configurations of Type 1 with a leading heavy edge $D$ such that $b(B)$ is a special vertex in $D$ and $B$ is a Type $B$ edge in this configuration. For every monochromatic heavy edge $B$, recolor $b(B)$ with color $j(B)+1$ (modulo $t$ ). By the choice of $b(B)$, we recolored only some high vertices.

We claim that the new coloring $f^{\prime \prime}$ does not have monochromatic heavy edges. Indeed, suppose that some heavy edge $D$ is monochromatic of color $j$ in $f^{\prime \prime}$. This means that it was not monochromatic of color $j$ in $f^{\prime}$, since in that case, a vertex of $R(D)$ would be recolored 


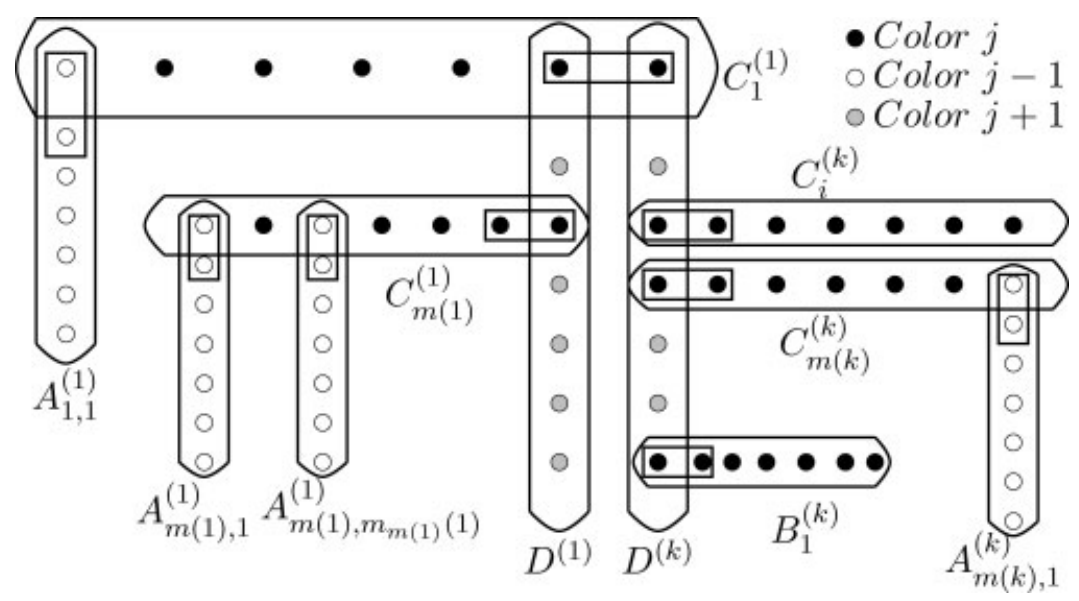

Fig. 2. An example of a Configuration of Type $2 b$.

to $j+1$. So, there are vertices $b_{1}, \ldots, b_{m^{\prime}}$ in $H(D)$ that were recolored from color $j-1$, and for each $b_{i}$, there is a heavy edge $B_{i}$ with $b_{i} \in R\left(B_{i}\right)$ that was monochromatic in $f^{\prime}$ and $b_{i}=b\left(B_{i}\right)$. So, we have a configuration of Type 1 in $f^{\prime}$ that contradicts the definition of the vertex $b\left(B_{1}\right)$.

Second recoloring: Let $C$ be a monochromatic edge of color $j(C)$ in the new coloring $f^{\prime \prime}$. By above, it is a light edge, and in $f^{\prime} C$ either was monochromatic of the same color, or some vertices $b_{1}, \ldots, b_{m^{\prime}} \in H(C)$ were of color $j(C)-1$, and each $b_{i}$ was in $R\left(B_{i}\right)$ for some heavy monochromatic edge $B_{i}$ and was recolored because of this edge. Suppose that for every $c \in$ $R(C)$, there is a configuration of Type 1 in coloring $f^{\prime \prime}$ with $m_{1}=m_{2}=\cdots=m_{m}=0=m^{\prime}$ and the leading edge containing $c$ as a special vertex, where $C$ is a Type $C$ edge. Then, each such configuration in $f^{\prime \prime}$ corresponds to some more general configuration of Type 1 in coloring $f^{\prime}$. It follows that we encounter a configuration of Type $2 \mathrm{~b}$ in $f^{\prime}$, a contradiction to the choice of $f^{\prime}$. Thus, every monochromatic edge $C$ in the new coloring $f^{\prime \prime}$ contains a vertex $c(C) \in R(C)$ such that there is no configuration of Type 1 in coloring $f^{\prime \prime}$ with the leading edge containing $c$ as a special vertex such that $m_{1}=m_{2}=\cdots=m_{m}=0=m^{\prime}$ and $C$ is a Type $C$ edge in this configuration.

For every monochromatic edge $C$ in $f^{\prime \prime}$, recolor $c(C)$ with color $j(C)+1$. Observe that at this second recoloring, we recolored only low vertices. Assume that some edge $D$ is monochromatic in the new coloring $f$ [of color $j(D)$ ]. If it was also monochromatic in $f^{\prime \prime}$, then $D$ is light, and some vertex of $R(D)$ would be recolored; so this is not the case. Thus, there are vertices $c_{1}, \ldots, c_{m}$ in $L(D)$ that were recolored from color $j(D)-1$, and for each $c_{i}$, there is a light edge $C_{i}$ with $c_{i} \in R\left(C_{i}\right)$ that was monochromatic in $f^{\prime \prime}$ of color $j(D)-1$ and $c_{i}=c\left(C_{i}\right)$. Furthermore, since $C_{i}$ was monochromatic in $f^{\prime \prime}$, either it also was monochromatic in $f^{\prime}$ or there are vertices $a_{i, 1}, \ldots, a_{i, m_{i}} \in H(C)$ of color $j(D)-2$ that were recolored in the first stage. In this case, in $f^{\prime}$ each $a_{i, i^{\prime}}$ was in $R\left(A_{i, i^{\prime}}\right)$ for some heavy monochromatic edge $A_{i, i^{\prime}}$ and was recolored in first stage because of this edge. Some vertices $b_{1}, \ldots, b_{m^{\prime}}$ in $H(D)$ also could be recolored in the first stage. Thus, we have a configuration of Type 1 in $f^{\prime}$, a contradiction to the choice of $c\left(C_{1}\right)$. As we recolored high vertices in the first stage and low at the second, no vertex is recolored more than once.

Thus, the theorem will be proved when we show that for every vertex $p$ in $\mathcal{H}$, the total contribution of configurations of Type 1 containing $p$ such that at least one of $m, m^{\prime}, m_{1}, \ldots, m_{m}^{\prime}$ 


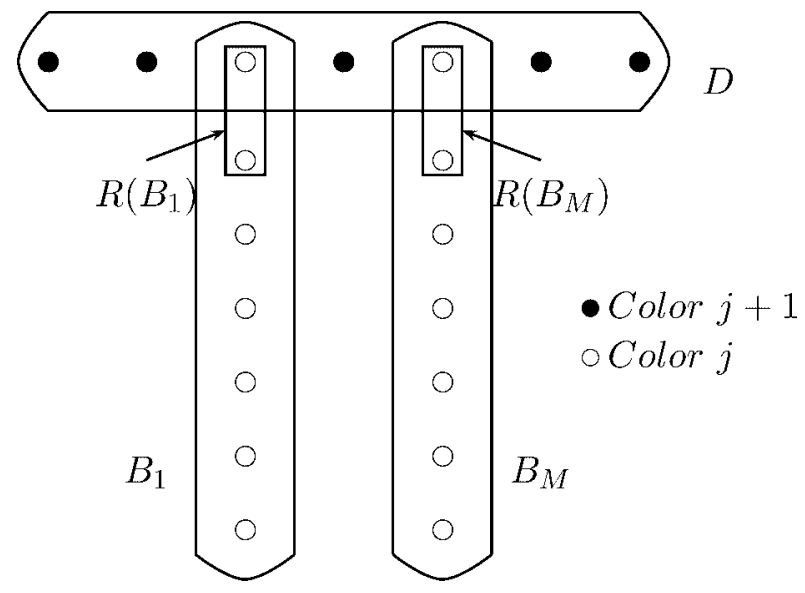

Fig. 3. An example of a Configuration of Type 1a.

exceeds $k$ is $o(1 / r)$ and that the total contribution of configurations of Types $2 \mathrm{a}$ and $2 \mathrm{~b}$ containing $p$ is $o(1 / r)$.

\subsection{Handling Configurations of Type 1}

We will first consider some partial cases.

2.4.1. Configuration of Type 1a. This is a configuration of Type 1 in which $m_{1}=m_{2}=$ $\cdots=m_{m}=0$ and $k \leq m+m^{\prime} \leq \frac{r}{10 b k}$.

For convenience of notation in handling configurations of Type 1a, define $B_{m^{\prime}+i}=C_{i}$ for $i=1, \ldots, m$ and let $M=m+m^{\prime}$. For $q=1, \ldots, M$, call edge $B_{q}$ determined if it intersects with $\cup_{i \leq q-1} B_{i}$ in at least $b+1$ vertices. Let $p \in V(\mathcal{H})$ and $z$ be a non-negative integer. Then the total contribution $\phi_{1 a}(p, M, z, D)$ of all configurations of Type 1a, containing $p$ such that $p \in D$ and exactly $z$ edges in $\left\{B_{1}, \ldots, B_{M}\right\}$ are determined is estimated as follows (Fig. 3):

$\left(\beta_{1}\right)$ The number of candidates for $D$ containing $p$ is at most $\operatorname{deg}(p) \leq t^{r} r$.

$\left(\beta_{2}\right)$ The number of ways to choose $b_{1}, \ldots, b_{M}$ in $D$ is at most $\left(\begin{array}{l}r \\ M\end{array}\right)$.

$\left(\beta_{3}\right)$ The number of choices of colors for vertices in $D$ such that vertices $b_{1}, \ldots, b_{M}$ are colored with $j$ and other are colored with $j+1$ is $t$.

$\left(\beta_{4}\right)$ The number of ways to choose which $z$ edges $B_{i}$ will be determined is $\left(\begin{array}{l}M \\ z\end{array}\right)$.

$\left(\beta_{5}\right)$ By Lemmas 6 and 7, the number of ways to choose a non-determined $B_{i}$ when we know the corresponding special vertex is at most $\frac{t^{r}}{r^{\epsilon}} \frac{4 k}{r}$.

$\left(\beta_{6}\right)$ Since every determined edge contains some $(b+1)$-tuple of vertices in the union of "previous" edges and these $(b+1)$-tuples should be distinct for different edges, the number of ways to choose a determined $B_{i}$ when we know the corresponding special vertex is at most $\left(\begin{array}{c}M r \\ b+1\end{array}\right)<\left(\begin{array}{c}r^{2} \\ b+1\end{array}\right)<r^{2 b+2}$.

$\left(\beta_{7}\right)$ Since

$$
\left|\bigcup_{l=1}^{i} B_{l}-\bigcup_{l=1}^{i-1} B_{l}\right| \geq \begin{cases}r-b, & \text { if } B_{i} \text { is non-determined } \\ r-M b, & \text { if } B_{i} \text { is determined, }\end{cases}
$$

and $M b \leq r / 10 k$, the size of each such configuration is at least $r+(M-z)(r-b)+z \frac{9 r}{10}$. 
Hence

$$
\phi_{1 a}(p, M, z, D) \leq t^{r} r t\left(\begin{array}{c}
r \\
M
\end{array}\right)\left(\begin{array}{c}
M \\
z
\end{array}\right)\left(\frac{4 k t^{r}}{r^{1+\epsilon}}\right)^{M-z} r^{2 z(b+1)}\left(\frac{r}{t(r-1)}\right)^{r+(M-z)(r-b)+0.9 z r} .
$$

Since $\left(\begin{array}{l}r \\ M\end{array}\right)\left(\begin{array}{l}M \\ z\end{array}\right) \leq r^{M}$ and $\left(\frac{r}{r-1}\right)^{r+(M-z)(r-b)+0.9 z r} \leq 3^{1+M}$, the last expression is at most

$$
3^{1+M}(4 k)^{M-z} t^{r+1+r(M-z)-r-(M-z)(r-b)-0.9 z r} r^{1+M-(M-z)(1+\epsilon)+2 z(b+1)} .
$$

Denoting the last expression by $\psi_{1 a}(M, z)$, we have

$$
\frac{\psi_{1 a}(M, z+1)}{\psi_{1 a}(M, z)} \leq \frac{1}{4 k} t^{-r+(r-b)-0.9 r} r^{(1+\epsilon)+2(b+1)}=\frac{1}{4 k} t^{-b-0.9 r} r^{2 b+3+\epsilon},
$$

which is less than $1 / 4$ for large $r$. Therefore,

$$
\begin{aligned}
\sum_{z=0}^{M} \phi_{1 a}(p, M, z, D)< & 2 \psi_{1 a}(M, 0) \\
& =3^{1+M} 2(4 k)^{M} t^{r+1+r M-r-M(r-b)} r^{1+M-M(1+\epsilon)}=6 \operatorname{tr}\left(\frac{12 k t^{b}}{r^{\epsilon}}\right)^{M} .
\end{aligned}
$$

Since for large $r, 12 k t^{b}<r^{\epsilon / 2}$, the last expression is less than $6 t r^{1-0.5 M \epsilon}$. Since $M \geq$ $k \geq \frac{20}{\epsilon}$, this is less than $6 t r^{-9}=o\left(r^{-8}\right)$. Thus, the total contribution $\phi_{1 a}(p, D)$ of all configurations of Type 1a such that $p \in D$ is less than

$$
\sum_{M=k}^{r} 2 \psi_{1 a}(M, 0)<r \cdot o\left(r^{-8}\right)=o\left(r^{-7}\right) .
$$

Now, we calculate the contribution of configurations of Type $1 a$ containing $p$ such that $p \notin D$. In this case, fix an edge $B_{i}$ containing $p$ in at most $t^{r} r$ ways. Then, we can choose vertex $b_{i} \in R\left(B_{i}\right)$ in at most $k$ ways and the edge $D$ containing $B_{i}$ in at most $k t^{r} r$ ways. To choose the remaining $M-1$ special vertices in $D$ there are only $\left(\begin{array}{c}r-1 \\ M-1\end{array}\right)$ ways. Then, using the same argument and almost the same calculations as earlier we get that the total contribution here is at most $k r$ times greater than $\sum_{M=1}^{r} 2 \psi_{1 a}(M, 0)$. Hence the total contribution, $\phi_{1 a}(p)$ of all configurations of Type la containing $p$ is $o\left(\frac{1}{r^{6}}\right)$.

2.4.2. Configuration of Type $1 \mathrm{~b}$. We need this structure to handle configurations of Type 1 in which $m_{1}=m_{2}=\cdots=m_{m}=0$ and $m+m^{\prime} \geq \frac{r}{10 b k}$. But we consider a somewhat different situation: it is a configuration of Type 1 in which $m_{1}=m_{2}=\cdots=m_{m}=0$ and $m+m^{\prime}=\left\lfloor\frac{r}{10 b k}\right\rfloor$, but non-special vertices in $D$ also allowed to be colored with color $j$ (and not only with $j+1)$. We will estimate the contributions of such new configurations.

As in case of Type 1a, define $B_{m^{\prime}+i}=C_{i}$ for $i=1, \ldots, m$ and let $M=m+m^{\prime}$.

For $p \in V(\mathcal{H})$ and an integer $z$, let $\phi_{1 b}(p, z, D)$ denote the total contribution of all configurations of Type $1 \mathrm{~b}$, containing $p$ such that $p \in D$ and exactly $z$ edges among $B_{i}$ are determined. We repeat the first half of the argument for Type 1a, replacing $\left(\beta_{3}\right)$ by the following:

$\left(\beta_{3}^{\prime}\right)$ The number of choices of colors for vertices in $D$ such that vertices $b_{1}, \ldots, b_{M}$ are colored with $j$ and other are colored with $j$ or $j+1$ is $t 2^{r-M}$. 
Because of the extra factor of $2^{r-M}$, instead of (13), we get

$$
\sum_{z=0}^{M} \phi_{1 b}(p, z, D)<6 t r\left(\frac{12 k t^{b}}{r^{\epsilon}}\right)^{M} 2^{r-M}
$$

Again, for large $r, 12 k t^{b}<r^{\epsilon / 2}$, and the last expression is at most $2^{r-M} 6 t r^{1-0.5 M \epsilon}$. Since $M=\left\lfloor\frac{r}{10 b k}\right\rfloor$ this is $o\left(r^{-8}\right)$.

Similarly, to the argument for configurations of Type 1a, the contribution of configurations of Type $1 \mathrm{~b}$ containing $p$ such that $p \notin D$ cannot exceed the last expression more than $r^{2}$ times. Thus, the total contribution $\phi_{1 a}(p)+\phi_{1 b}(p)$ of all configurations of Types 1a and $1 \mathrm{~b}$ containing $p$ is $o\left(r^{-6}\right)$.

From now on, we consider only $t$-colorings of $V(\mathcal{H})$ such that no configurations of types 1a or $1 \mathrm{~b}$ occur.

2.4.3. Configuration of Type 1c. This is a configuration of Type 1 in which $k \leq$ $m+m^{\prime} \leq \frac{r}{5 b k}$.

By $\left(\alpha_{8}\right)$ and $\left(\alpha_{9}\right)$ in the definition configurations of Type 1 , for every $i=1, \ldots, m$, the set $C_{i} \cup \bigcup_{i^{\prime}=1}^{m_{i}} A_{i^{\prime}}$ with our coloring form a configuration of Type $1 \mathrm{a}$ or $1 \mathrm{~b}$ if $m_{i} \geq k$. Since such configurations are forbidden, we assume that $m_{i}<k$ for every $i$. Similarly, if $m^{\prime} \geq \frac{r}{10 b k}$, then the set $B_{1} \cup \ldots B_{m^{\prime}} \cup D$ with our coloring forms a configuration of Type $1 \mathrm{~b}$, and so, we consider only the case $m^{\prime}<\frac{r}{10 b k}$.

To calculate carefully the contributions of configurations of Type $1 \mathrm{c}$, let $\hat{m}_{i}$ denote the number of edges in $\left\{A_{i, 1}, \ldots, A_{i, m_{i}}\right\}$ that are distinct from all $A_{l, l^{\prime}}$ for all $l<i$.

Let $p \in V(\mathcal{H}), k \leq m+m^{\prime} \leq \frac{r}{5 b k}$, and $z, z^{\prime}, \hat{m}_{1}, \ldots, \hat{m}_{m}$ be non-negative integers. Let $\hat{M}=\hat{m}_{1}+\cdots+\hat{m}_{m}$. Let $\phi_{1 c}\left(p, m^{\prime}, m, \hat{m}_{1}, \ldots, \hat{m}_{m}, z, z^{\prime}, D\right)$ denote the total contribution of all configurations of Type 1c with parameters $m^{\prime}, m, \hat{m}_{1}, \ldots, \hat{m}_{m}$ containing $p$ such that $p \in D$, exactly $z$ edges among $B_{1}, \ldots, B_{m^{\prime}}, C_{1}, \ldots, C_{m}$ are determined, and exactly $z^{\prime}$ other edges are determined. We can estimate it as follows:

$\left(\gamma_{1}\right)$ The number of candidates for $D$ containing $p$ is at $\operatorname{most} \operatorname{deg}(p) \leq t^{r} r$.

$\left(\gamma_{2}\right)$ The number of ways to choose $b_{1}, \ldots, b_{m^{\prime}}$ and $c_{1}, \ldots, c_{m}$ in $D$ is at most $\left(\begin{array}{c}r \\ m+m^{\prime}\end{array}\right)\left(\begin{array}{c}m+m^{\prime} \\ m\end{array}\right)$.

$\left(\gamma_{3}\right)$ The number of choices of colors for vertices in $D$ such that vertices $b_{1}, \ldots, b_{m^{\prime}}$ and $c_{1}, \ldots, c_{m}$ are colored with $j$ and all others are colored with $j+1$ is $t$.

$\left(\gamma_{4}\right)$ The number of ways to choose which $z$ edges among $B_{1}, \ldots, B_{m^{\prime}}, C_{1}, \ldots, C_{m}$ will be determined is $\left(\begin{array}{c}m^{\prime}+m \\ z\end{array}\right)$.

$\left(\gamma_{5}\right)$ By Lemmas 6 and 7, the number of ways to choose a non-determined $B_{i}$ when we know $b_{i} \in R\left(B_{i}\right)$ or $C_{i}$ when we know $c_{i} \in R\left(C_{i}\right)$ is at most $\frac{t^{r}}{r \epsilon} \frac{4 k}{r}$.

$\left(\gamma_{6}\right)$ The number of ways to choose a determined edge $B_{i}$ when we know $b_{i} \in R\left(B_{i}\right)$ or $C_{i}$ when we know $c_{i} \in R\left(C_{i}\right)$ is at most $\left(\begin{array}{c}\left(m+m^{\prime}\right) r \\ b+1\end{array}\right)<\left(\begin{array}{c}r^{2} \\ b+1\end{array}\right)<r^{2 b+2}$.

$\left(\gamma_{7}\right)$ The number of ways to choose all vertices $a_{i, i^{\prime}}$ in $C_{1} \cup \ldots C_{m}$ that will be colored with $j-1$ is at most $\left(\begin{array}{c}m r \\ \hat{M}\end{array}\right)$.

$\left(\gamma_{8}\right)$ The number of ways to choose which $z^{\prime}$ edges among $A_{i, i^{\prime}}$ will be determined is $\left(\begin{array}{l}\hat{M} \\ z^{\prime}\end{array}\right)$.

$\left(\gamma_{9}\right)$ The number of ways to choose a non-determined $A_{i, i^{\prime}}$ when we know $a_{i, i^{\prime}} \in R\left(A_{i, i^{\prime}}\right)$ is at most $\frac{t^{r}}{r^{\epsilon}} \frac{4 k}{r}$.

$\left(\gamma_{10}\right)$ The number of ways to choose a determined edge $A_{i, i^{\prime}}$ when we know $a_{i, i^{\prime}} \in R\left(A_{i, i^{\prime}}\right)$ is at most $\left(\begin{array}{c}m r k \\ b+1\end{array}\right)<\left(\begin{array}{c}r^{2} \\ b+1\end{array}\right)<r^{2 b+2}$. 
$\left(\gamma_{11}\right)$ To estimate the size of such a configuration, recall that $m^{\prime}+m \leq \frac{r}{5 b k}$ and that $\hat{m}_{i} \leq m_{i} \leq$ $k-1$ for each $i$. Therefore, $m^{\prime}+m+\hat{M} \leq k\left(m^{\prime}+m\right) \leq \frac{r}{5 b}$. Similarly to (12), when we add edges one by one to the configuration, every non-determined edge adds at least $r-b$ vertices and every determined edge adds at least $r-b\left(m^{\prime}+m+\hat{M}\right) \geq r-r / 5=4 r / 5$ vertices to the union. It follows that the size of each such configuration is at least $r+\left(m+m^{\prime}+\hat{M}-z-z^{\prime}\right)(r-b)+\left(z+z^{\prime}\right) \frac{4 r}{5}$.

Hence, $\phi_{1 c}\left(p, m^{\prime}, m, \hat{m}_{1}, \ldots, \hat{m}_{m}, z, z^{\prime}, D\right)$ is at most

$$
\begin{aligned}
& t^{r} r\left(\begin{array}{c}
r \\
m+m^{\prime}
\end{array}\right)\left(\begin{array}{c}
m+m^{\prime} \\
m
\end{array}\right) t\left(\begin{array}{c}
m^{\prime}+m \\
z
\end{array}\right)\left(\prod_{i=1}^{m}\left(\begin{array}{c}
r \\
\hat{m}_{i}
\end{array}\right)\right)\left(\begin{array}{c}
\hat{M} \\
z^{\prime}
\end{array}\right) \\
& \quad \times\left(\frac{4 k t^{r}}{r^{1+\epsilon}}\right)^{m+m^{\prime}+\hat{M}-z-z^{\prime}} r^{\left(z+z^{\prime}\right)(2 b+2)}\left(\frac{r}{t(r-1)}\right)^{r+\left(m+m^{\prime}+\hat{M}\right)(r-b)-\left(z+z^{\prime}\right)(0.2 r-b)} .
\end{aligned}
$$

Since $\frac{r}{r-1}<3^{1 / r}$ and

$$
\begin{aligned}
& \left(\begin{array}{c}
r \\
m+m^{\prime}
\end{array}\right)\left(\begin{array}{c}
m+m^{\prime} \\
m
\end{array}\right)\left(\begin{array}{c}
m^{\prime}+m \\
z
\end{array}\right)\left(\prod_{i=1}^{m}\left(\begin{array}{c}
r \\
\hat{m}_{i}
\end{array}\right)\right)\left(\begin{array}{c}
\hat{M} \\
z^{\prime}
\end{array}\right) \\
& \leq r^{m+m^{\prime}}\left(m+m^{\prime}\right)^{z} r^{\hat{M}} \hat{M}^{z^{\prime}} \leq r^{m+m^{\prime}+\hat{M}+z+z^{\prime}},
\end{aligned}
$$

$\phi_{1 c}\left(p, m^{\prime}, m, \hat{m}_{1}, \ldots, \hat{m}_{m}, z, z^{\prime}, D\right)$ is at most

$$
\begin{aligned}
& t^{r+1+r\left(m+m^{\prime}+\hat{M}-z-z^{\prime}\right)-r-\left(m+m^{\prime}+\hat{M}\right)(r-b)+\left(z+z^{\prime}\right)(0.2 r-b)}(4 k)^{m+m^{\prime}+\hat{M}-z-z^{\prime}} \\
& \quad \times r^{1+m+m^{\prime}+\hat{M}+z+z^{\prime}-(1+\epsilon)\left(m+m^{\prime}+\hat{M}-z-z^{\prime}\right)+\left(z+z^{\prime}\right)(2 b+2)} 3^{1+m+m^{\prime}+\hat{M}} \\
& =t^{1+b\left(m+m^{\prime}+\hat{M}\right)-\left(z+z^{\prime}\right)(0.8 r+b)}(4 k)^{m+m^{\prime}+\hat{M}-z-z^{\prime}} r^{1-\epsilon\left(m+m^{\prime}+\hat{M}\right)+\left(z+z^{\prime}\right)(2 b+4+\epsilon)} 3^{1+m+m^{\prime}+\hat{M}} .
\end{aligned}
$$

Denoting the last expression by $\psi_{1 c}\left(m+m^{\prime}+\hat{M}, z+z^{\prime}\right)$, we have

$$
\frac{\psi_{1 c}\left(m+m^{\prime}+\hat{M}, z+z^{\prime}+1\right)}{\psi_{1 c}\left(m+m^{\prime}+\hat{M}, z+z^{\prime}\right)} \leq \frac{1}{4 k} t^{-0.8 r-b} r^{2 b+4+\epsilon},
$$

which is less than $1 / 4 r$ for large $r$. Therefore,

$$
\sum_{z=0}^{m^{\prime}+m} \sum_{z^{\prime}=0}^{\hat{M}} \phi_{1 c}\left(p, m^{\prime}, m, \hat{m}_{1}, \ldots, \hat{m}_{m}, z, z^{\prime}, D\right)<2 \psi_{1 c}\left(m+m^{\prime}+\hat{M}, 0\right)=6 t r\left(\frac{12 k t^{b}}{r^{\epsilon}}\right)^{m+m^{\prime}+\hat{M}} .
$$

Observe that the last bound depends only on $\hat{M}$ and not on the values of particular $\hat{m}_{1}, \ldots, \hat{m}_{m}$. Let

$$
\phi_{1 c}\left(p, m^{\prime}, m, \hat{M}, D\right)=\sum_{\left(\hat{m}_{1}, \ldots, \hat{m}_{m}\right): \hat{m}_{1}+\cdots+\hat{m}_{m}=\hat{M}} \sum_{z+z^{\prime}=0}^{m+m^{\prime}+\hat{M}} \phi_{1 c}\left(p, m^{\prime}, m, \hat{m}_{1}, \ldots, \hat{m}_{m}, z, z^{\prime}, D\right) .
$$

Random Structures and Algorithms DOI 10.1002/rsa 
Since the number of $m$-tuples $\left(\hat{m}_{1}, \ldots, \hat{m}_{m}\right)$ with $\hat{m}_{1}+\cdots+\hat{m}_{m}=\hat{M}$ is $\left(\begin{array}{c}m+\hat{M}-1 \\ m-1\end{array}\right)<2^{m+\hat{M}}$, for large $r$, by (14),

$$
\phi_{1 c}\left(p, m^{\prime}, m, \hat{M}, D\right) \leq 6 t r\left(\frac{12 k t^{b}}{r^{\epsilon}}\right)^{m+m^{\prime}+\hat{M}} 2^{m+\hat{M}} \leq 6 t r^{1-0.5 \epsilon\left(m+m^{\prime}+\hat{M}\right)} .
$$

Since $m+m^{\prime} \geq k \geq \frac{20}{\epsilon}$, the last expression is $o\left(r^{-8}\right)$. Since $m^{\prime}<k, m<r / k$, and $\hat{M}<m k<r$, the total contribution of all configurations of Type 1c containing $p$ such that $p \in D$, is at most

$$
o\left(r^{-8}\right) k \frac{r}{k} r=o\left(r^{-6}\right) .
$$

Similarly, to the case of configurations of Type 1a, the total contribution of all configurations of Type 1c containing $p$ such that $p \in B_{i}$, is at most $r^{2}$ times greater than our bound above. The bound for the total contribution of all configurations of Type 1c containing $p$ such that $p$ is in a light edge $C_{i}$ or in $A_{i, i^{\prime}}$ is only $k$ times greater than the bound above, since $R\left(C_{i}\right)$ and $R\left(A_{i, i^{\prime}}\right)$ consist only of low vertices. Hence, the total contribution, $\phi_{1 c}(p)$, of all configurations of Type 1c containing $p$ is $o\left(r^{-4}\right)$.

2.4.4. Configuration of Type $1 d$. We need it to handle configurations of Type 1 in which $m+m^{\prime} \geq \frac{r}{5 b k}$. Since the situation with $m^{\prime} \geq \frac{r}{10 b k}$ is covered by configurations of Type $1 \mathrm{~b}$, it is enough to consider the following situation: Configuration of Type $1 \mathrm{~d}$ is a configuration of Type 1 in which $m=\left\lfloor\frac{r}{10 b k}\right\rfloor$ and $m^{\prime}=0$ but non-special vertices in $D$ also allowed to be colored with color $j$. We will estimate the contributions of such configurations.

Let $p \in V(\mathcal{H})$, and $z, z^{\prime}, \hat{m}_{1}, \ldots, \hat{m}_{m}$ be non-negative integers. Let $\hat{M}=\hat{m}_{1}+\cdots+\hat{m}_{m}$. Let $\phi_{1 d}\left(p, \hat{m}_{1}, \ldots, \hat{m}_{m}, z, z^{\prime}, D\right)$ denote the total contribution of all configurations of Type $1 \mathrm{~d}$ with parameters $\hat{m}_{1}, \ldots, \hat{m}_{m}$ containing $p$ such that $p \in D$, exactly $z$ edges among $C_{1}, \ldots, C_{m}$ are determined, and exactly $z^{\prime}$ other edges are determined. The ingredients for an upper bound on $\phi_{1 d}\left(p, \hat{m}_{1}, \ldots, \hat{m}_{m}, z, z^{\prime}, D\right)$ are almost the same as for $\phi_{1 c}\left(p, m^{\prime}, m, \hat{m}_{1}, \ldots, \hat{m}_{m}, z, z^{\prime}, D\right)$ above with $m^{\prime}=0$; the only difference is that Item $\left(\gamma_{3}\right)$ is replaced with.

$\left(\gamma_{3}^{\prime}\right)$ The number of choices of colors for vertices in $D$ such that vertices $c_{1}, \ldots, c_{m}$ are colored with $j$ and all others are colored either with $j$ or with $j+1$ is $t 2^{r-m}$.

Thus, repeating the argument for configurations of Type 1c, instead of (14), we will obtain

$$
\sum_{z=0}^{m} \sum_{z^{\prime}=0}^{\hat{M}} \phi_{1 d}\left(p, \hat{m}_{1}, \ldots, \hat{m}_{m}, z, z^{\prime}, D\right)<2^{r-m} 6 t r\left(\frac{12 k t^{b}}{r^{\epsilon}}\right)^{m+\hat{M}} .
$$

Since $m=\left\lfloor\frac{r}{10 b k}\right\rfloor$, the extra factor of $2^{r-m}$ does not hurt our upper bounds, and we essentially repeat the argument from (14) above for configurations of Type 1c.

Forbidding configurations of Types $1 c$ and $1 d$ forbids all configurations of Type 1 with $m+m^{\prime} \geq k$.

\subsection{Handling Configurations of Type 2}

2.5.1. Configuration of Type $2 a$. Let $j \in\{1,2, \ldots, t\}$. Suppose that there exist $k$ configurations of Type 1 a (for the same $j$ ) with edge sets (for $l=1, \ldots, k)\left\{D^{(l)}, B_{1}^{(l)}, \ldots, B_{m^{\prime}(l)}^{(l)}\right\}$ such that 
i. $B_{1}^{(1)}=B_{1}^{(2)}=\cdots=B_{1}^{(k)}$,

ii. all $b_{1}^{(1)}, b_{1}^{(2)}, \ldots, b_{1}^{(k)}$ are distinct vertices, so that $\left\{b_{1}^{(1)}, b_{1}^{(2)}, \ldots, b_{1}^{(k)}\right\}=R\left(B_{1}^{(1)}\right)$,

iii. all edges $D^{(l)}$ and $B_{i}^{(l)}$ are heavy.

Then the union of these $k$ configurations is a configuration of Type 2a.

It is possible that $D^{(l)}=D^{\left(l^{\prime}\right)}$ for $l \neq l^{\prime}$, but in this case, (since both $b_{1}^{(l)}$ and $b_{1}^{\left(l^{\prime}\right)}$ are colored with $j$ ) $b_{1}^{(l)}$ coincides with some $b_{i}^{\left(l^{\prime}\right)}$ such that $B_{i}^{\left(l^{\prime}\right)}$ is distinct from $B_{1}^{(1)}$. Thus, in any case, there are at least $k$ distinct edges among $D^{(l)}$ and $B_{i}^{(l)}$. On the other hand, since large configurations of Type 1a are forbidden, $m^{\prime}$ and each of $m^{\prime}(l)$ is at most $k$. So, the total number of involved edges is at most $(k+1)^{2}$. Since we have so few edges, in calculations we will not care about determined edges, our only concern will be repetitions of edges.

Given a configuration of Type $2 \mathrm{a}$, let $x$ denote the number of distinct $D^{(l)}$. Order the edges of our configuration so that the first edge is $B_{1}^{(1)}$ followed by all of the $D^{(l)}$, and then all the other edges. With a given ordering, for all suitable $l$, let $\hat{m}(l)$ denote the number of corresponding edges that do not appear earlier in the order. Let $M=\sum_{l=1}^{k} \hat{m}(l)$.

Let $p \in V(\mathcal{H})$. Let $\Phi=\phi_{2 b}\left(p, \hat{m}(1), \ldots, \hat{m}(k), x, B_{1}^{(1)}\right)$ denote the total contribution of all configurations of Type $2 \mathrm{a}$ with the corresponding given parameters containing $p$ such that $p \in B_{1}^{(1)}$.

We can estimate $\Phi$ as follows:

$\left(\delta_{1}\right)$ The number of candidates for $B_{1}^{(1)}$ containing $p$ is at most $t^{r} r$.

$\left(\delta_{2}\right)$ The number of partitions of $R\left(B_{1}^{(1)}\right)$ into $x$ non-empty sets is less than $k^{x}$.

$\left(\delta_{3}\right)$ The number of ways to choose for every of the $x$ parts in the partition an edge containing this class is at most $\left(t^{r} r^{-\epsilon}\right)^{x}$, since the number of heavy edges containing any given vertex is at most $t^{r} r^{-\epsilon}$. These edges will be our edges $D^{(1)}, \ldots, D^{(k)}$.

$\left(\delta_{4}\right)$ The number of choices of color $j$ is $t$.

$\left(\delta_{5}\right)$ The number of ways to choose for every $l \in\{1, \ldots, k\}$, vertices $b_{1}(l), \ldots, b_{\hat{m}(l)}(l)$ is at most $\prod_{l=1}^{k}\left(\begin{array}{c}r \\ \hat{m}(l)\end{array}\right) \leq\left(\begin{array}{c}k r \\ M\end{array}\right) \leq(k r)^{M}$.

$\left(\delta_{6}\right)$ By Lemma 7, the number of ways to choose a $B_{i}^{(l)}$ when we know $b_{i}(l) \in R\left(B_{i}^{(l)}\right)$ is at most $\frac{t^{r}}{r^{\epsilon}} \frac{4 k}{r}$.

$\left(\delta_{7}\right)$ To estimate the size of such a configuration, recall that in total we have at most $(k+1)^{2}$ edges. Therefore, each edge has at most $(k+1)^{2} b$ vertices that are common with any other edge. It follows that the size of each such configuration is at least $\left(r-(k+1)^{2} b\right)(1+x+M)$.

Hence

$$
\begin{aligned}
\Phi & \leq t^{r} r\left(\frac{k t^{r}}{r^{\epsilon}}\right)^{x} t(k r)^{M}\left(\frac{4 k t^{r}}{r^{1+\epsilon}}\right)^{M}\left(\frac{r}{t(r-1)}\right)^{\left(r-(k+1)^{2} b\right)(1+x+M)} \\
& \leq k^{x+M+M} 4^{M} r^{1-\epsilon x+M-(1+\epsilon) M} t^{r+r x+1+r M-\left(r-(k+1)^{2} b\right)(1+x+M)} 3^{1+x+M} \\
& =k^{x+2 M} 4^{M} r^{1-\epsilon(x+M)} t^{1+(k+1)^{2} b(1+x+M)} 3^{1+x+M} \leq t r^{1+\epsilon}\left(\frac{12 k^{2} t^{(k+1)^{2} b}}{r^{\epsilon}}\right)^{1+x+M} .
\end{aligned}
$$

The number of different presentations of $M$ in the form $M=\sum_{l=1}^{k} \hat{m}(l)$ is at $\operatorname{most}\left(\begin{array}{c}M+k-1 \\ M\end{array}\right)<$ $2^{M+k-1}$. Therefore, the total contribution, $\phi_{2 b}\left(p, x, M, B_{1}^{(1)}\right)$, of all configurations of Type $2 \mathrm{a}$ with given $x$ and $M$ containing $p$ such that $p \in B_{1}^{(1)}$ for large $r$ is at most 


$$
2^{M+k-1} \operatorname{tr}^{1+\epsilon}\left(\frac{12 k^{2} t^{(k+1)^{2} b}}{r^{\epsilon}}\right)^{1+x+M} \leq 2^{k-x} \operatorname{tr}^{1+\epsilon-0.5 \epsilon(1+x+M)}
$$

By construction, $M+x \geq k$. Hence, since $k \geq 0.20 / \epsilon$,

$$
\phi_{2 a}\left(p, x, M, B_{1}^{(1)}\right) \leq 2^{k} t r^{-8}=o\left(r^{-7}\right) .
$$

Since $x \leq k$ and $M \leq k^{2}$, the total contribution of all configurations of Type 2a containing $p$ such that $p \in B_{1}^{(1)}$ is also $o\left(r^{-7}\right)$. The total contribution of all configurations of Type $2 \mathrm{a}$ containing $p$ such that $p \in D^{(l)}$ for some $l$ is estimated in practically the same steps and also is $o\left(r^{-7}\right)$. The same holds for the total contribution of all configurations of Type $2 \mathrm{a}$ containing $p$ such that $p \in B_{i}^{(l)}$ for some $l$ and $i$. Thus, the total contribution, $\phi_{2 a}(p)$, of all configurations of Type 2a containing $p$ is $o\left(r^{-6}\right)$.

2.5.2. Configuration of Type 2b. Let $j \in\{1,2, \ldots, t\}$. Suppose that there exist $k$ configurations of Type $1 \mathrm{c}$ (for the same $j$ ) with edge sets (for $l=1, \ldots, k$ )

$$
\left\{D^{(l)}, B_{1}^{(l)}, \ldots, B_{m^{\prime}(l)}^{(l)}, C_{1}^{(l)}, \ldots, C_{m(l)}^{(l)}, A_{1,1}^{(l)}, \ldots, A_{1, m_{1}(l)}^{(l)}, \ldots, A_{m(l), m_{m(l)}(l)}^{(l)}\right\}
$$

such that $C_{1}^{(1)}=C_{1}^{(2)}=\cdots=C_{1}^{(k)}$ and all $c_{1}^{(1)}, c_{1}^{(2)}, \ldots, c_{1}^{(k)}$ are distinct vertices, so that $\left\{c_{1}^{(1)}, c_{1}^{(2)}, \ldots, c_{1}^{(k)}\right\}=R\left(C_{1}^{(1)}\right)$. Then the union of these $k$ configurations is a configuration of Type $2 b$. As in configurations of Type 1c, some representative vertices can coincide, in which case the corresponding edges also should coincide.

It is possible that $D^{(l)}=D^{\left(l^{\prime}\right)}$ for $l \neq l^{\prime}$, but in this case, (since both $c_{1}^{(l)}$ and $c_{1}^{\left(l^{\prime}\right)}$ are colored with $j) c_{1}^{(l)}$ coincides with some $c_{i}^{\left(l^{\prime}\right)}$ such that $C_{i}^{\left(l^{\prime}\right)}$ is distinct from $C_{1}^{(1)}$. Thus, in any case, there are at least $k$ distinct edges among $D^{(l)}$ and $C_{i}^{(l)}$. On the other hand, since large configurations of Type 1c are forbidden, each of $m(l), m^{\prime}(l), m_{i}(l)$ is at most $k$. So, the total number of involved edges is at most $k(k+1)$.

Given a configuration of Type $2 \mathrm{~b}$, let $x$ denote the number of distinct $D^{(l)}$. Order the edges of our configuration so that first is listed the edge $C_{1}^{(1)}$, then all $D^{(l)}$, then all $B_{i}^{(l)}$ (in any order), then all $C_{i}^{(l)}$, and then all other edges. With a given ordering, for all suitable $i$ and $l$, let $\hat{m}(l), \hat{m}^{\prime}(l)$, and $\hat{m}_{i}(l)$ denote the number of corresponding edges that do not appear earlier in the order. Let $M=\sum_{l=1}^{k}\left(\hat{m}(l)+\hat{m}^{\prime}(l)\right)$ and

$$
\hat{M}=\sum_{l=1}^{k} \sum_{i=1}^{m} \hat{m}_{i}(l)
$$

Let $p \in V(\mathcal{H})$. Let

$$
\Phi=\phi_{2 b}\left(p, \hat{m}^{\prime}(1), \ldots, \hat{m}^{\prime}(k), \hat{m}(1), \ldots, \hat{m}(k), \hat{m}_{1}(1), \ldots, \hat{m}_{1}(k), \ldots, \hat{m}_{m}(k), x, C_{1}^{(1)}\right)
$$

denote the total contribution of all configurations of Type $2 \mathrm{~b}$ with the corresponding parameters containing $p$ such that $p \in C_{1}^{(1)}$.

We can estimate $\Phi$ as follows:

$\left(\kappa_{1}\right)$ The number of candidates for $C_{1}^{(1)}$ containing $p$ is at most $t^{r} r$.

$\left(\kappa_{2}\right)$ The number of partitions of $R\left(C_{1}^{(1)}\right)$ into $x$ non-empty sets is less than $k^{x}$. 
$\left(\kappa_{3}\right)$ The number of ways to choose for every of the $x$ parts in the partition an edge containing this class is at most $\left(t^{r} r^{-\epsilon}\right)^{x}$, since every vertex in $R\left(C_{1}^{(1)}\right)$ is a low vertex. These edges will be our edges $D^{(1)}, \ldots, D^{(k)}$.

$\left(\kappa_{4}\right)$ The number of choices of color $j$ is $t$.

$\left(\kappa_{5}\right)$ The number of ways to choose for every $l \in\{1, \ldots, k\}$, vertices $b_{1}(l), \ldots, b_{\hat{m}^{\prime}(l)}(l)$ and $c_{2}(l), \ldots, c_{\hat{m}(l)}(l)$ in $D^{(l)}$ is at most $\prod_{l=1}^{k}\left(\begin{array}{c}r \\ \hat{m}(l)+\hat{m}^{\prime}(l)\end{array}\right)\left(\begin{array}{c}\hat{m}(l)+\hat{m}^{\prime}(l) \\ \hat{m}^{\prime}(l)\end{array}\right)$.

$\left(\kappa_{6}\right)$ By Lemmas 6 and 7, the number of ways to choose a $B_{i}^{(l)}$ when we know $b_{i}(l) \in R\left(B_{i}^{(l)}\right)$ or $C_{i}^{(l)}$ when we know $c_{i}(l) \in R\left(C_{i}^{(l)}\right)$ is at most $\frac{t^{r}}{r^{\epsilon}} \frac{4 k}{r}$.

$\left(\kappa_{7}\right)$ The number of ways to choose all vertices $a_{i, i^{\prime}}(l)$ in $\cup_{l=1}^{k} \cup_{i=1}^{\hat{m}(l)} C_{i}^{(l)}$ that will be colored with $j-1$ is at most $\left(\begin{array}{c}k^{2} r \\ \hat{M}\end{array}\right)$.

$\left(\kappa_{8}\right)$ The number of ways to choose an $A_{i, i^{\prime}}^{(l)}$ when we know $a_{i, i^{\prime}}(l) \in R\left(A_{i, i^{\prime}}^{(l)}\right)$ is at most $\frac{t^{r}}{r^{\epsilon}} \frac{4 k}{r}$.

$\left(\kappa_{9}\right)$ To estimate the size of such a configuration, recall that in total we have at most $(k+1)^{3}$ edges. Therefore, each edge has at most $(k+1)^{3} b$ vertices that are common with any other edge. It follows that the size of each such configuration is at least $\left(r-(k+1)^{3} b\right)(1+x+M+\hat{M})$.

Hence

$$
\begin{aligned}
\Phi \leq t^{r} r\left(\frac{k t^{r}}{r^{\epsilon}}\right)^{x} t \prod_{l=1}^{k}\left(\begin{array}{c}
r \\
\hat{m}(l)+\hat{m}^{\prime}(l)
\end{array}\right)\left(\begin{array}{c}
\hat{m}(l)+\hat{m}^{\prime}(l) \\
\hat{m}^{\prime}(l)
\end{array}\right)\left(\begin{array}{c}
k^{2} r \\
\hat{M}
\end{array}\right)\left(\frac{4 k t^{r}}{r^{1+\epsilon}}\right)^{M+\hat{M}} \\
\times\left(\frac{r}{t(r-1)}\right)^{\left(r-(k+1)^{3} b\right)(1+x+M+\hat{M})} .
\end{aligned}
$$

Since $\left(\frac{r}{r-1}\right)^{\left(r-(k+1)^{3} b\right)(1+x+M+\hat{M})} \leq 3^{1+x+M+\hat{M}}$ and

$$
\prod_{l=1}^{k}\left(\begin{array}{c}
r \\
\hat{m}(l)+\hat{m}^{\prime}(l)
\end{array}\right)\left(\begin{array}{c}
\hat{m}(l)+\hat{m}^{\prime}(l) \\
\hat{m}^{\prime}(l)
\end{array}\right)\left(\begin{array}{c}
k^{2} r \\
\hat{M}
\end{array}\right) \leq r^{M}\left(k^{2} r\right)^{\hat{M}},
$$

we have

$$
\begin{aligned}
\Phi & \leq k^{x+M+3 \hat{M}} 4^{M+\hat{M}} r^{1-x \epsilon-\epsilon(M+\hat{M})} t^{r+r x+1+r(M+\hat{M})-\left(r-(k+1)^{3} b\right)(1+x+M+\hat{M})} 3^{1+x+M+\hat{M}} \\
& =k^{x+M+3 \hat{M}} 4^{M+\hat{M}} r^{1-\epsilon(x+M+\hat{M})}\left(3 t^{(k+1)^{3} b}\right)^{1+x+M+\hat{M}} \leq r^{1+\epsilon}\left(\frac{12 k^{3} t^{(k+1)^{3} b}}{r^{\epsilon}}\right)^{1+x+M+\hat{M}} .
\end{aligned}
$$

The last bound does not depend on values of $\hat{m}(l), \hat{m}^{\prime}(l)$, and $m_{i}(l)$, but only on $x, M$, and $M^{\prime}$. The number of different presentations of $M$ in the form $M=\sum_{l=1}^{k}\left(\hat{m}(l)+\hat{m}^{\prime}(l)\right)$ is at most $\left(\begin{array}{c}M+2 k-1 \\ M\end{array}\right)<2^{M+2 k-1}$. Similarly, the number of different presentations of $\hat{M}$ in the form (16) is at most the number of different presentations of $\hat{M}$ as a sum of at most $k^{2}$ nonnegative summands, which is at most

$$
\sum_{q=1}^{k^{2}}\left(\begin{array}{c}
\hat{M}+q \\
q
\end{array}\right) \leq \sum_{q=1}^{k^{2}}\left(\begin{array}{c}
\hat{M}+k^{2} \\
q
\end{array}\right) \leq 2^{k^{2}+\hat{M}}
$$


Therefore, the total contribution, $\phi_{2 b}\left(p, x, M, \hat{M}, C_{1}^{(1)}\right)$, of all configurations of Type $2 \mathrm{~b}$ with given $x, M$, and $\hat{M}$ containing $p$ such that $p \in C_{1}^{(1)}$ is at most

$$
2^{M+2 k-1} 2^{k^{2}+\hat{M}} r^{1+\epsilon}\left(\frac{12 k^{3} t^{(k+1)^{3} b}}{r^{\epsilon}}\right)^{1+x+M+\hat{M}}<2^{(k+1)^{2}} r^{1+\epsilon}\left(\frac{12 k^{3} t^{(k+1)^{3} b}}{r^{\epsilon}}\right)^{1+x+M+\hat{M}}
$$

For large $r$, this does not exceed $2^{(k+1)^{2}} r^{1+\epsilon-0.5 \epsilon(1+x+M+\hat{M})}$. As observed earlier, $x+M \geq k \geq$ $20 / \epsilon$. Thus for large $r, \phi_{2 b}\left(p, x, M, \hat{M}, C_{1}^{(1)}\right)=o\left(r^{-8}\right)$. Since $x \leq k, M \leq 2 k^{2}$, and $\hat{M} \leq k^{3}$, the total contribution, of all configurations of Type 2 b containing $p$ such that $p \in C_{1}^{(1)}$ is also $o\left(r^{-8}\right)$. Similarly, to the argument for configurations of Type 1c, the total contribution, of all configurations of Type $2 \mathrm{~b}$ containing $p$ such that $p \in D(l)$, or $p \in B_{i}(l)$, or $p \in C_{i}(l)$, or $p \in A_{i, i^{\prime}}(l)$ does not exceed the obtained bound more than $r^{2}$ times. Thus for large $r$, the total contribution, $\phi_{2 b}(p)$, of all configurations of Type $2 \mathrm{~b}$ containing $p$ is $o\left(r^{-6}\right)$.

\section{LOWER BOUNDS ON THE NUMBER OF EDGES}

\subsection{Trimming}

To get lower bound on the number of edges in an $r$-uniform $(t+1)$-chromatic simple hypergraph, Erdős and Lovász [2] applied a simple but quite useful technique of trimming. A trimming of a hypergraph $\mathcal{H}$ is the hypergraph $F(\mathcal{H})$ obtained from $\mathcal{H}$ by deleting from each edge a vertex of maximum possible degree. Trimming has two useful properties: (a) if $\mathcal{H}$ is not $t$-colorable, then $F(\mathcal{H})$ also is; and (b) if $\mathcal{H}$ is simple and $F(\mathcal{H})$ has a vertex of degree at least $d$, then $\mathcal{H}$ has at least $d+1$ vertices of degree at least $d$. We will somewhat elaborate upon the notion of trimming.

For positive integers $x$ and $D$, an edge $A$ of a hypergraph $\mathcal{H}$ is $(x, D)$-heavy, if at least $x$ vertices in $A$ have degree at least $D$ in $\mathcal{H}$. An $(x, D)$-trimming of a hypergraph $\mathcal{H}$ is the hypergraph $F_{x, D}(\mathcal{H})$ obtained from $\mathcal{H}$ in two steps: first choose in each edge $A$ a vertex $a(A)$ that is contained in the most $(x, D)$-heavy edges; then replace each edge $A$ with $A-a(A)$. The ordinary trimming above can be considered as a $(1,1)$-trimming.

Let $F_{x, D}^{(m)}(\mathcal{H})$ denote the hypergraph obtained from $\mathcal{H}$ by applying $(x, D)$-trimming $m$ times.

Lemma 8. Let $b, x, y, d, s$, and $D$ be positive integers and $\mathcal{H}$ be a hypergraph.

a. If $\mathcal{H}$ is b-simple, $F_{x, D}^{(b)}(\mathcal{H})$ has a vertex that belongs to at least $d(x, D)$-heavy edges and $\mathcal{H}$ has $y$ vertices that belong to at least $d(x, D)$-heavy edges each, then $\left(\begin{array}{l}y \\ b\end{array}\right) \geq d$.

b. If $\mathcal{H}$ has girth at least $2 s+1, b \leq s$, and $F_{x, D}^{(b)}(\mathcal{H})$ has a vertex that belongs to at least $d(x, D)$-heavy edges, then $\mathcal{H}$ has at least $(d-1)^{b}$ vertices at distance exactly $b$ from $v$ that belong to at least $d(x, D)$-heavy edges each.

Proof. For convenience, denote $F_{x, D}^{(0)}(\mathcal{H})=\mathcal{H}$. By definition, every edge $A \in E(\mathcal{H})$ contains distinct vertices $a^{(1)}(A), \ldots, a^{(b)}(A)$ such that for $i=1, \ldots, b$,

$$
E\left(F_{x, D}^{(i)}(\mathcal{H})\right)=\left\{A-\left\{a^{(1)}(A), \ldots, a^{(i)}(A)\right\}: A \in E(\mathcal{H})\right\}
$$

and vertex $a^{(i)}(A)$ is contained in the most of $(x, D)$-heavy edges of the hypergraph $F_{x, D}^{(i-1)}(\mathcal{H})$ among the vertices in $A^{(i-1)}:=A-\left\{a^{(1)}(A), \ldots, a^{(i-1)}(A)\right\}$. 
Suppose that $\mathcal{H}$ is $b$-simple and $v$ is a vertex in $F_{x, D}^{(b)}(\mathcal{H})$ that belongs to at least $d(x, D)$ heavy edges. Suppose that the edges $A_{1}^{(b)}, \ldots, A_{d}^{(b)}$ of $F_{x, D}^{(b)}(\mathcal{H})$ contain $v$. By the definition of $(x, D)$-trimming, each of the vertices $a^{(b)}\left(A_{1}\right), \ldots, a^{(b)}\left(A_{d}\right)$ is contained in at least $d(x, D)$ heavy edges in $F_{x, D}^{(b-1)}(\mathcal{H})$ (otherwise, $v$ would be the corresponding $a^{(b)}\left(A_{i}\right)$ ). Similarly, each of the vertices $a^{(b-1)}\left(A_{1}\right), \ldots, a^{(b-1)}\left(A_{d}\right)$ is contained in at least $d(x, D)$-heavy edges in $F_{x, D}^{(b-2)}(\mathcal{H})$, and so on.

Let $Y$ be the set of vertices in $\mathcal{H}$ that are contained in at least $d(x, D)$-heavy edges. By the previous paragraph, each of the vertices $a^{(j)}\left(A_{i}\right)$ for $i=1, \ldots, d$ and $j=1, \ldots, b$ is in $Y$. Vertices $a^{(j)}\left(A_{i}\right)$ and $a^{\left(j^{\prime}\right)}\left(A_{i^{\prime}}\right)$ may coincide for distinct $i$ and $i^{\prime}$, but the sets $\left\{a^{(1)}\left(A_{i}\right), \ldots, a^{(b)}\left(A_{i}\right)\right\}$ should be distinct for distinct $i$, since $\mathcal{H}$ is $b$-simple. Thus, the number of $b$-element subsets of $Y$ is at least $d$. This proves (a).

Suppose now that the girth of $\mathcal{H}$ is at least $2 s+1, b \leq s$, and $v$ is a vertex in $F_{x, D}^{(b)}(\mathcal{H})$ that belongs to at least $d(x, D)$-heavy edges. Suppose that the edges $A_{1}^{(b)}, \ldots, A_{d}^{(b)}$ of $F_{x, D}^{(b)}(\mathcal{H})$ contain $v$. As above, each of the vertices $a^{(b)}\left(A_{1}\right), \ldots, a^{(b)}\left(A_{d}\right)$ is contained in at least $d(x, D)$-heavy edges in $F_{x, D}^{(b-1)}(\mathcal{H})$. Moreover, since the girth of $\mathcal{H}$ is at least three, all $a^{(b)}\left(A_{1}\right), \ldots, a^{(b)}\left(A_{d}\right)$ are distinct and each of them is a neighbor of $v$. Thus, each of $a^{(b)}\left(A_{i}\right)$ is contained in some $d(x, D)$-heavy edges $A_{i, 1}^{(b-1)}, \ldots, A_{i, d}^{(b-1)}$. If $b=1$, then we are done.

Suppose $b \geq 2$. Then the girth of $\mathcal{H}$ is at least five and for each $1 \leq i \leq d$, exactly one edge among $A_{i, 1}^{(b-1)}, \ldots, A_{i, d}^{(b-1)}$ (namely, $A_{i}^{(b)}$ ) contains $v$, and all others are almost disjoint amongst them and are disjoint from all other $A_{j, 1}^{(b-1)}, \ldots, A_{j, d}^{(b-1)}$ for $j \neq i$. It follows that for all $i_{1}, i_{2}=1, \ldots, d$ such that $A_{i_{1}, i_{2}}^{(b-1)} \neq A_{i_{1}}^{(b)}$, all vertices $a^{(b-1)}\left(A_{i_{1}, i_{2}}^{(b-1)}\right)$ are distinct and each such $a^{(b-1)}\left(A_{i_{1}, i_{2}}^{(b-1)}\right)$ belongs to at least $d(x, D)$-heavy edges $A_{i_{1}, i_{2}, 1}^{(b-2)}, \ldots, A_{i_{1}, i_{2}, d}^{(b-2)}$ in $F_{x, D}^{(b-2)}(\mathcal{H})$ and is at distance 2 from $v$ in $\mathcal{H}$. In particular, there are at least $d(d-1)$ of them. Again, if $b=2$, then we are done. Otherwise the girth of $\mathcal{H}$ is at least seven and for all triples $\left(i_{1}, i_{2}, i_{3}\right)$ such that $A_{i_{1}, i_{2}, i_{3}}^{(b-2)} \neq A_{i_{1}, i_{2}}^{(b-1)}$, the sets $A_{i_{1}, i_{2}, i_{3}}^{(b-2)}-a^{(b-1)}\left(A_{i_{1}, i_{2}}^{(b-1)}\right)$ are disjoint from each other and from all edges $\left(j_{1}, j_{2}, j_{3}\right)$ for $\left(j_{1}, j_{2}\right) \neq\left(i_{1}, i_{2}\right)$.

Continuing in this way, finally, we construct $d(d-1)^{b-1}$ distinct vertices $a^{(1)}\left(A_{i_{1}, i_{2}, \ldots, i_{b}}\right)$ such that each of them belongs to at least $d(x, D)$-heavy edges in $F_{x, D}^{(0)}(\mathcal{H})$ and is at distance exactly $b$ from $v$.

\subsection{Size of $(t+1)$-Chromatic b-Simple Hypergraphs}

Theorem 9. Let $t$ and $b$ be positive integers, $\epsilon>0$, and $r$ be sufficiently large in comparison with $t, b$, and $\epsilon$. Let $\mathcal{H}$ be $a(t+1)$-chromatic $r$-uniform $b$-simple hypergraph. Then $\mathcal{H}$ has at least $t^{r(1+1 / b)} r^{-\epsilon}$ edges.

Proof. Let $x=\lceil(r-b) / 2\rceil$ and $D=\left\lceil t^{r-b} / r^{\epsilon / 3}\right\rceil$. Let $\mathcal{H}_{1}=F_{1,1}^{(b)}(\mathcal{H})$. By construction, $\mathcal{H}_{1}$ is $(r-b)$-uniform and $b$-simple. Since $\mathcal{H}$ is not $t$-colorable, $\mathcal{H}_{1}$ is also not $t$-colorable. So, by Theorem 4, either

i. $\mathcal{H}_{1}$ has a vertex of degree at least $t^{r-b}(r-b)$, or

ii. $\mathcal{H}_{1}$ has a vertex contained in at least $D(x, D)$-heavy edges.

If (i) holds, then by Lemma $8(a), \mathcal{H}$ has at least $\left(t^{r-b}(r-b)\right)^{1 / b}$ vertices of degree at least $t^{r-b}(r-b)$. Hence the number of edges in $\mathcal{H}$ is at least

$$
\frac{1}{r}\left(t^{r-b}(r-b)\right)^{1+1 / b} \geq t^{r(1+1 / b)} .
$$


Suppose now that (ii) holds. Let $Y$ be the set of vertices of degree at least $D$ in $\mathcal{H}_{1}$. Each $(x, D)$-heavy edge containing $v$ intersects $Y-v$ in at least $x-1$ vertices. No $b$-tuple of vertices of $Y-v$ is contained in more than one edge containing $v$. Therefore,

$$
\left(\begin{array}{c}
|Y| \\
b
\end{array}\right) \geq D\left(\begin{array}{c}
x-1 \\
b
\end{array}\right)
$$

For large $r$, this implies $|Y|^{b} \geq D(r / 3)^{b}$, so that the number of edges in $\mathcal{H}_{1}$ is at least

$$
\frac{1}{r} D^{1+1 / b} \frac{r}{3} \geq \frac{1}{3}\left(\frac{t^{r-b}}{r^{\epsilon / 3}}\right)^{1+1 / b} \geq t^{r(1+1 / b)} r^{-\epsilon}
$$

\subsection{Size of $(t+1)$-Chromatic Hypergraphs of Girth $2 s+1$ and $2 s+2$}

Theorem 10. Let $t$ and $s$ be positive integers, $\epsilon>0$, and $r$ be sufficiently large in comparison with $t, s$, and $\epsilon$. Let $\mathcal{H}$ be a $(t+1)$-chromatic $r$-uniform hypergraph of girth at least $2 s+1$. Then $\mathcal{H}$ has at least $t^{r(1+s)} r^{-\epsilon}$ edges. Moreover, if the girth of $\mathcal{H}$ is at least $2 s+2$, then $\mathcal{H}$ has at least $t^{r(1+s)} r^{1-\epsilon}$ edges.

Proof. Let $x=\lceil(r-2 s+1) / 2\rceil$ and $D=\left\lceil t^{r-2 s+1} / r^{\epsilon / 3 s}\right\rceil$. Let $\mathcal{H}_{1}=F_{1,1}^{(s)}(\mathcal{H})$ and $\mathcal{H}_{2}=$ $F_{x, D}^{(s-1)}\left(\mathcal{H}_{1}\right)$. By construction, $\mathcal{H}_{2}$ is $(r-2 s+1)$-uniform. Since $\mathcal{H}$ is not $t$-colorable, $\mathcal{H}_{2}$ is also not $t$-colorable. So, by Theorem 4 , either

i. $\mathcal{H}_{2}$ has a vertex of degree at least $t^{r-2 s+1}(r-2 s+1)$, or

ii. $\mathcal{H}_{2}$ has a vertex contained in at least $D(x, D)$-heavy edges.

If (i) holds, then $\mathcal{H}_{1}$ also has a vertex of degree at least $t^{r-2 s+1}(r-2 s+1)$. By Lemma $8(\mathrm{~b}), \mathcal{H}$ has at least $\left(t^{r-2 s+1}(r-2 s+1)-1\right)^{s}$ vertices of degree at least $t^{r-2 s+1}(r-2 s+1)$. Hence, the number of edges in $\mathcal{H}$ is at least

$$
\frac{1}{r}\left(t^{r-2 s+1}(r-2 s+1)-1\right)^{1+s} \geq t^{r(1+s)} r .
$$

Suppose now that (ii) holds. By Lemma $8(\mathrm{~b}), \mathcal{H}_{1}$ contains a set $F(s, v)$ of at least $(D-1)^{s-1}$ vertices at distance exactly $s-1$ from $v$ such that each of them is contained in at least $D$ $(x, D)$-heavy edges. Since the girth of $\mathcal{H}_{1}$ is at least $2 s+1$, each $u \in F(s, v)$ is contained in exactly one edge $M(u)$ on the unique shortest path from $u$ to $v$. Also, if for $u \in F(s, v)$ an edge $A(u) \neq M(u)$ meets or coincides with any edge containing any vertex $w$ at distance at most $s-1$ from $v$, then $\mathcal{H}_{1}$ contains a cycle of length at most $2 s$, a contradiction.

Thus, we have a set $F^{\prime}(s, v)$ of at least $(D-1)^{s}(x, D)$-heavy edges such that each edge in $F^{\prime}(s, v)$ contains exactly one of our $(D-1)^{s-1}$ special vertices at distance $s-1$ from $v$ and no other vertices at distance at most $s-1$ from $v$. This means that each of the edges in $F^{\prime}(s, v)$ contains at least $x-1$ vertices of degree at least $D$ that do not belong to any other edge in $F^{\prime}(s, v)$. Since $x$ is about $r / 2$ and $r$ is much larger than $s$ and $t$, it follows that the number of edges in $\mathcal{H}_{1}$ is at least

$$
\frac{1}{r}(D-1)^{s+1}(x-1) \geq \frac{1}{3} D^{s+1} \geq \frac{1}{3}\left(\frac{t^{r-2 s+1}}{r^{\epsilon / 3 s}}\right)^{1+s} \geq t^{r(1+s)} r^{-\epsilon} .
$$


This proves the statement for girth $2 s+1$.

Suppose that the girth of $\mathcal{H}$ is at least $2 s+2$. It (i) holds, then the statement is already proved above. Suppose that (ii) holds. Then we construct $F^{\prime}(s, v)$ exactly as in the previous paragraph. Consider an edge $A \in F^{\prime}(s, v)$ and any vertex $z \in A$ of degree at least $D$ that does not belong to other edges in $F^{\prime}(s, v)$. If any of the at least $D-1$ distinct from $A$ edges containing $z$ contains also a vertex from any other edge in $F^{\prime}(s, v)$, then $\mathcal{H}$ has a cycle of length $2 s+1$ or less. Thus, all these edges are distinct and the total number of them is at least

$$
\left|F^{\prime}(s, v)\right|(x-1)(D-1) \geq \frac{r}{3} D^{s+1} \geq \frac{r}{3}\left(\frac{t^{r-2 s+1}}{r^{\epsilon / 3 s}}\right)^{1+s} \geq t^{r(1+s)} r^{1-\epsilon} .
$$

\section{UPPER BOUND ON $f(r, t, b)$}

The Erdôs-Lovász bound (1) can be easily extended to $b$-simple hypergraphs as follows.

Theorem 11. If $b \geq 1$ and $t \geq 2$ are fixed and $r$ is sufficiently large, then

$$
f(r, t, b) \leq 10 t^{2}\left(2 t^{r} r^{2}\right)^{(b+1) / b} .
$$

Proof. We follow the lines of the proof of Theorem 1' in [2] by Erdôs and Lovász.

Let

$$
n=\left\lceil 4 t\left(2 t^{r} r^{2(b+1)}\right)^{1 / b}\right\rceil \text { and } \quad m=4 n t^{r+1} \sim 8 t^{2}\left(2 t^{r} r^{2}\right)^{(b+1) / b} .
$$

We let $\mathcal{H}_{0}$ be the edgeless hypergraph with $\left|V\left(\mathcal{H}_{0}\right)\right|=t n$ and for $i=1, \ldots, m$ will obtain $\mathcal{H}_{i}$ from $\mathcal{H}_{i-1}$ by adding an edge $e_{i}$ so that

a. $\mathcal{H}_{i}$ remains $b$-simple and

b. $x_{i} \leq\left(1-1 / 4 t^{r}\right) x_{i-1}$, where $x_{i}$ is the number of $n$-element subsets of $V\left(\mathcal{H}_{0}\right)=V\left(\mathcal{H}_{i}\right)$ not containing edges of $\mathcal{H}_{i}$.

As in [2], if we manage (a) and (b) until $i=m$, then

$$
x_{m} \leq x_{0}\left(1-\frac{1}{4 t^{r}}\right)^{m}=\left(\begin{array}{c}
t n \\
n
\end{array}\right)\left(1-\frac{1}{4 t^{r}}\right)^{4 n t^{r+1}} \leq \frac{(t e)^{n}}{e^{t n}}=\left(\frac{t e}{e^{t}}\right)^{n}<1 .
$$

Suppose that (a) and (b) hold for $i=0,1, \ldots, j$. Let $S$ be an $n$-element subset of $V\left(\mathcal{H}_{j}\right)$ not containing any edge of $\mathcal{H}_{j}$. If an $r$-tuple $R \subset S$ cannot be added to $\mathcal{H}_{j}$ because (a) would fail, then $R$ has $b+1$ elements in common with some $e_{i}, i \leq j$. The number of such $R \subset S$ is at most

$$
j\left(\begin{array}{c}
r \\
b+1
\end{array}\right)\left(\begin{array}{l}
n-b-1 \\
r-b-1
\end{array}\right) \leq \frac{m r^{b+1}}{(b+1) !}\left(\begin{array}{l}
n \\
r
\end{array}\right) \frac{r^{b+1}}{(n-b)^{b+1}}=\frac{m r^{2(b+1)}}{(b+1) !(n-b)^{b+1}}\left(\begin{array}{l}
n \\
r
\end{array}\right) .
$$

By (17), for fixed $b$ and $t$ and for large $r$, we have

$$
m \frac{r^{2(b+1)}}{(b+1) !(n-b)^{b+1}} \leq 4 n t^{r+1} \frac{2 r^{2(b+1)}}{(b+1) ! n^{b+1}} \leq 4 t^{r+1} \frac{2 r^{2(b+1)}}{2 !(4 t)^{b} 2 t^{r} r^{2(b+1)}} \leq \frac{1}{2} .
$$


It follows that every $n$-element $S \subset V\left(\mathcal{H}_{j}\right)$ not containing any edge of $\mathcal{H}_{i}$ contains at least $0.5\left(\begin{array}{l}n \\ r\end{array}\right)$ candidates for $e_{j+1}$. Therefore, some $r$-element subset $e_{j+1}$ of $V\left(\mathcal{H}_{i}\right)$ is a candidate for at least

$$
x_{j} \cdot 0.5\left(\begin{array}{l}
n \\
r
\end{array}\right)\left(\begin{array}{c}
t n \\
r
\end{array}\right)^{-1} \geq \frac{x_{j} n(n-1) \cdots(n-r+1)}{2(t n)^{r}} .
$$

$n$-element subsets of $V\left(\mathcal{H}_{j}\right)$ not containing any edge of $\mathcal{H}_{j}$. Since $n \geq 8 r^{2}$,

$$
\frac{x_{j} n(n-1) \cdots(n-r+1)}{2(t n)^{r}} \geq \frac{x_{j}}{2 t^{r}}\left(\frac{n-r+1}{n}\right)^{r} \geq \frac{x_{j}}{4 t^{r}} .
$$

Thus, if we choose this $e_{j+1}$, then (a) and (b) hold for $i=j+1$.

\section{COMMENTS}

1. Although all $b, t, s$, and $\epsilon$ are considered fixed, they also can be viewed as very slowly growing functions of $r$. For example, it is possible to consider $\epsilon=c \frac{\log \log \log r}{\log \log r}$ for a small positive constant $c$.

2. Condition (7) in the definition of $(t, \epsilon)$-sparse $r$-uniform hypergraphs can be weakened by any polynomial factor of $r$. The problem in sharpening our results is in (8).

3. The proofs of Theorems 4 and 3 and inequalities (4) and (5) can be adapted to list coloring. In particular, the following statement holds (and implies the other results).

Theorem 12. If $b \geq 1, t \geq 2$, and $\epsilon>0$ are fixed and $r$ is sufficiently large, then every $r$-uniform b-simple $(t, \epsilon)$-sparse hypergraph $\mathcal{H}$ is list $t$-colorable.

We start from a random coloring $f$ of vertices of $\mathcal{H}$ where each vertex $v$ is colored with a color $f(v)$ uniformly at random chosen from its list List $(v)$ independently from all other vertices. To adapt the proof of Theorem 4, for each vertex $v \in V(\mathcal{H})$, fix any bijection $v_{v}$ of the list $\operatorname{List}(v)$ onto itself with $v_{v}(\alpha) \neq \alpha$ for each $\alpha \in \operatorname{List}(v)$. In all recolorings

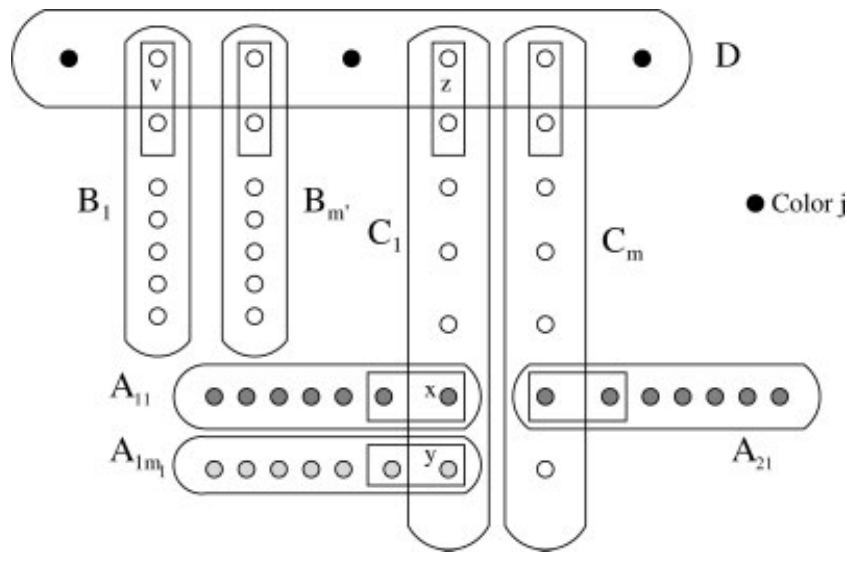

Fig. 4. Configuration of Type 1 for list colorings. 
during the proof, each vertex $v$ of color $j$ will be tried to be recolored (if at all) into the color $v_{v}(j)$ (instead of color $j+1$, as it was in the proof in Section 2). So, the Configuration of Type 1 in Fig. 1 will look more like in Fig. 4. In this picture, if the "main color" of the edge $D$ is $j$, then $f(v)=v_{v}^{-1}(j), f(z)=v_{z}^{-1}(j), f(x)=v_{x}^{-1}(f(z))=v_{x}^{-1}\left(v_{z}^{-1}(j)\right)$, $f(y)=v_{y}^{-1}(f(z))=v_{y}^{-1}\left(v_{z}^{-1}(j)\right)$, and so on. So, the colors of vertices $v$ and $z$ (likewise, of $x$ and $y$ ) can be different, but the structure remains the same, and for each vertex, only one color is "dangerous" for the configuration. Similarly, we define the other configurations. After these definitions and before any recoloring is done, all the calculations will be the same as in Section 2, and the result follows.

\section{ACKNOWLEDGMENT}

The authors thank both referees for helpful comments.

\section{REFERENCES}

[1] J. Beck, A remark concerning arithmetic progressions, J Combin Theory Ser A 29 (1980), 376-379.

[2] P. Erdős and L. Lovász, Problems and results on 3-chromatic hypergraphs and some related questions, Infinite and finite sets, Colloquia Math Soc János Bolyai 10 (1973), 609-627.

[3] Z. Szabó, An application of Lovász' local lemma-A new lower bound for the van der Waerden number, Random Structures Algorithms 1 (1990), 343-360. 